\title{
INTERNAL DYNAMICS OF THE DWARF ELLIPTICAL NGC 185
}

\author{
ENRICO V. HELD \\ Osservatorio Astronomico di Bologna, Via Zamboni 33, I-40126, Bologna, Italy \\ TIM DE ZeEUW \\ Sterrewacht Leiden, Postbus 9513, 2300 RA Leiden, The Netherlands \\ Jeremy Mould and Alain Picard \\ Palomar Observatory, Caltech 105-24, Pasadena, California 91125 \\ Received 1 August 1991; revised 11 October 1991
}

\begin{abstract}
Kinematic observations along the major and minor axis of the E2 dwarf elliptical galaxy NGC 185 are presented. The velocity dispersion is constant at $28 \pm 8 \mathrm{~km} \mathrm{~s}^{-1}$ between $3^{\prime \prime}$ and $40^{\prime \prime}$, but may increase to about twice that value in the center. We find an upper limit of $10 \mathrm{~km} \mathrm{~s}^{-1}$ for the rotation along either axis, so that the velocity distribution is anisotropic. The derived $\mathscr{C} / L_{B}$ is $\sim 3$ in solar units. Analysis of the kinematic and photometric data now available shows that giant ellipticals and dwarfs fall on a continuous sequence in the $(L, \sigma)$ plane.
\end{abstract}

\section{INTRODUCTION}

Models of the formation and evolution of diffuse dwarf elliptical galaxies $(\mathrm{dE})$ require detailed knowledge of their structural properties and internal dynamics. While modern CCD detectors allow an accurate derivation of structural parameters and colors for even such low surface brightness objects, spectroscopic observation of the internal dynamics of dwarfs remains a challenging task. Until recently, dwarf ellipticals were tacitly expected to be supported by rotation, by extrapolating from the kinematic behavior of normal elliptical galaxies (gE) (Davies et al. 1983). Contrary to this expectation, NGC 205, a dwarf companion of the Andromeda Nebula, turned out to be a slow rotator, which is supported by an anisotropic velocity distribution (Carter \& Sadler 1990; Held et al. 1990, henceforth Paper I). Paltoglou \& Freeman (1987) drew a similar conclusion for the faint local dwarf Fornax, a result confirmed by Mateo et al. (1991b). Most recently, Bender et al. (1991, hereafter referred to as BPN) confirmed previous results on NGC 205, and presented new rotation and velocity dispersion measurements of two more M31 companions, NGC 185 and NGC 147. All of the three objects appear to be anisotropic systems. Lack of rotational support might well be evidence that dwarf ellipticals are triaxial.

Bender \& Nieto (1990) studied the internal kinematics of a sample of low-luminosity normal ellipticals $\left(M_{B}>-18\right)$, and found that objects of relatively low surface brightness are supported by an anisotropic velocity distribution, whereas faint ellipticals with relatively high surface brightness follow the trend of brighter galaxies measured by Davies et al. (1983). It should be noted, however, that only one object in Bender \& Nieto's (1990) sample was previously classified as a genuine dwarf elliptical.

In an effort to understand the internal dynamics of verylow-mass systems, we undertook a kinematic study of the next logical candidate, the Local Group dwarf elliptical galaxy NGC 185, for which no kinematic data were available at the time of the observations. Given the difficulties inherent in measuring rotation and velocity dispersion of such lowsurface-brightness objects, it is important to have independent measurements of the kinematics of dE's. At a distance of about $75 \mathrm{kpc}$ from M31, NGC 185 is likely not to be affected by dynamical interactions with its bigger neighbor. NGC 185 appears bluer in the nucleus due to a young stellar population, even though there are no clusters of young stars as large as those observed in NGC 205 (Hodge 1963; Price 1985). The existence of a modest central Population I component was also demonstrated by the Very Large Array (VLA) observations of Johnson \& Gottesman (1983), which indicated the presence of $\sim 1.5 \times 10^{5} \mathscr{M}_{\odot}$ of $\mathrm{H}$ I, with a distribution peaked at about $30^{\prime \prime}$ from the center. This is consistent with other evidence of ongoing or recent star formation activity. Faint $\mathrm{CO}$ emission was detected by Wiklind \& Rydbeck (1986), indicating the presence of $\sim 10^{6} \mathscr{M}_{\odot}$ of molecular gas.

In this paper we report spectroscopic observations of NGC 185, and discuss some implications of the available data on kinematic parameters of dwarf elliptical galaxies. In Sec. 2 we describe the observations and data reduction techniques. Our kinematic results for NGC 185 are presented and discussed in Sec. 3, and in Sec. 4 we describe parameter correlations for dwarf ellipticals as a class.

\section{OBSERVATIONS AND' REDUCTION}

NGC 185 was observed on 24-27 August 1990 with the Double Spectrograph (Oke \& Gunn 1982) at the $5 \mathrm{~m}$ Hale telescope. Only the blue spectra were reduced. The blue arm detector was an $800 \times 800$ TI CCD with an extended blue response. For most of the spectra, four pixels were binned together along the spatial direction, in order to improve the signal-to-noise ( $\mathrm{S} / \mathrm{N})$ ratio. The resulting spatial scale was 1.67 arcsec pixel ${ }^{-1}$. The only exception was a major-axis spectrum, for which double binning was used to achieve a better spatial resolution in the nucleus. The 1200 line grating and $1^{\prime \prime} \times 120^{\prime \prime}$ slit yielded a sampling $0.56 \AA$ pixel $^{-1}$, and a resolution $\sim 1.5 \AA \mathrm{FWHM}$, in the spectral range $\lambda \lambda 3800$ $4250 \AA$ ( $\lambda \lambda 3960-4410 \AA$ for the double-binned spectrum) Four $1^{\mathrm{h}}$ spectra of the galaxy major axis were taken at a slit position angle $50^{\circ}$. The nucleus was placed near one end of the slit, to obtain kinematic data as far as possible from the galaxy center. The spectrograph was then rotated at the position angle $230^{\circ}$, and four additional $1^{\mathrm{h}}$ exposures were obtained of the same region of the galaxy, with the nucleus 
again suitably offset. Three $75^{\mathrm{m}}$ exposures of the minor axis were also taken at P.A. $=140^{\circ}$. Finally, the exposure for the double-binned spectrum was $1^{\mathrm{h}}$ at P.A. $=50^{\circ}$. Comparison spectra of an iron hollow-cathode lamp were taken before and after each exposure. Template stars of spectral types F$G$, to be compared with the galaxy spectra by a Fourier method, were also observed.

Data reduction followed the same procedure as in Paper I. The pedestal level of the CCD frames turned out to be variable during the night, possibly because of a recent preflash treatment. This caused initial problems with flat fielding. These were solved by subtracting the bias level as derived from an unexposed region of each frame. After the standard trimming and flat-fielding steps, cosmic rays hits were removed by a median filtering of pixels above a fixed threshold. The spectra were then line-by-line wavelength calibrated, and rebinned onto a logarithmic wavelength scale using the routines of the Caltech/AAO FIGARO package. The curvature correction was good, the residual distortion being of the order one-tenth of pixel, or $\pm 5 \mathrm{~km} \mathrm{~s}^{-1}$ (as estimated from the shape of sky emission lines on the corrected frames). The spectra were also corrected for a slight misalignment of the chip, and shifted so that the nucleus fell at the same position in all of the frames. Finally, high $\mathrm{S} / \mathrm{N}$ spectra were then obtained by coadding all spectra observed with the same instrumental setup. A sky spectrum was extracted from a region in each frame far from the galaxy nucleus. The contribution of galaxy light at that location, although not negligible, was less critical than for NGC 205, because of the lower mean surface brightness of NGC 185 (Kent 1987).

Radial velocity $(v)$ and dispersion $(\sigma)$ profiles were measured using the Fourier fitting program developed by Franx (Franx et al. 1989). Data were binned along the spatial direction so as to give $\sim 10^{5}$ photons in each bin. The spectra of NGC 185 were compared with those of template stars in the wavelength interval $\lambda \lambda 4000-4230 \AA$, thus excluding from the fit the $\mathrm{Ca}$ II $H$ and $K$ lines, which are known to give systematically biased velocity dispersions. For the single double-binned major-axis spectrum, the wavelength range $\lambda \lambda 4000-4400 \AA$ was used, which contains $H_{\gamma}, H_{\delta}$, the $G$ band, and many $\mathrm{Ca}$ I and $\mathrm{Fe} \mathrm{I}$ lines. Test runs using the entire observed spectral interval showed the derived radial velocities to be essentially independent of the adopted wavelength range. As a second-order refinement, the residual curvature of sky emission lines in the distortion-corrected frames was utilized to correct the radial velocity profiles.

Tests were made to ensure the reliability of our results against possible systematic errors arising from inadequate sky subtraction. As in Paper I, realistic artificial spectra were constructed using the NGC 185 luminosity profile (Kent 1987), a rotation curve rising to $40 \mathrm{~km} \mathrm{~s}^{-1}$ at $120^{\prime \prime}$ from the galaxy nucleus, and velocity dispersion in the range $20-80 \mathrm{~km} \mathrm{~s}^{-1}$. We then applied the same reduction steps as followed for the NGC 185 spectra. In particular, a "sky" was extracted from the outer rows and subtracted from the entire spectrum. This implies that some galaxy light from the outer region was subtracted from each row of the spectrum. These experiments showed that the velocity curve could be recovered out to $40^{\prime \prime}$ from the nucleus, with only a slight shift in systemic velocity, of the order of $10 \mathrm{~km} \mathrm{~s}^{-1}$. The velocity dispersion could be recovered with $10 \%$ accuracy for input values larger than $20 \mathrm{~km} \mathrm{~s}^{-1}$, and again $r<40^{\prime \prime}$. Beyond this limit in radius, however, the residual intensity after "sky" subtraction becomes too low for the Fourier program to derive meaningful kinematic information.

\section{RESULTS}

In Fig. 1 we present the velocity and dispersion profiles of NGC 185. The rotation curves along the major and minor axis of the galaxy are shown in Figs. 1(a) and 1(c), respectively. The results from different coadded spectra are in excellent agreement, and show no evidence of a gradient along either axis. Fitting a straight line to all major-axis data yields an upper limit of the order $10 \mathrm{~km} \mathrm{~s}^{-1}$ over a radius range of $40^{\prime \prime}$, which is mainly set by the estimated uncertainty on the distortion correction. Our measurement of the heliocentric velocity gives a redshift of $-217 \pm 8 \mathrm{~km} \mathrm{~s}^{-1}$. This value is intermediate between those of Sandage \& Tammann (1981) $\left(-227 \mathrm{~km} \mathrm{~s}^{-1}\right)$ and BPN $\left(-202 \mathrm{~km} \mathrm{~s}^{-1}\right)$.

The velocity dispersion measurements show a similarly good agreement, and the resulting profiles are displayed in Figs. 1(b) and 1(d). The median velocity dispersions obtained from the data points in the range 0 " -30 " derived from the two coadded spectra along the major and minor axes, are $27.3 \pm 7.2(\mathrm{rms})$ and $29.1 \pm 9.6 \mathrm{~km} \mathrm{~s}^{-1}$, respectively. Along the major axis, the velocity dispersion of NGC 185 appears essentially constant out to 40 " from the center. Central velocity dispersions from the double-binned major-axis spectrum are presented in Fig. 2. Because of the improved spatial resolution of this spectrum, the derived $\sigma$ profile shows a fairly convincing increase up to $60-70 \mathrm{~km} \mathrm{~s}^{-1}$ in the central 2-3" (7-10 pc). This increase might also be due to the influence of broad Balmer lines of young stars on the measured velocity dispersion. In our spectra, the regions excluding Balmer lines are too limited to allow meaningful measurements. Therefore this possibility cannot be entirely ruled out on the basis of our data.

Formal errors for the kinematic measurements provided

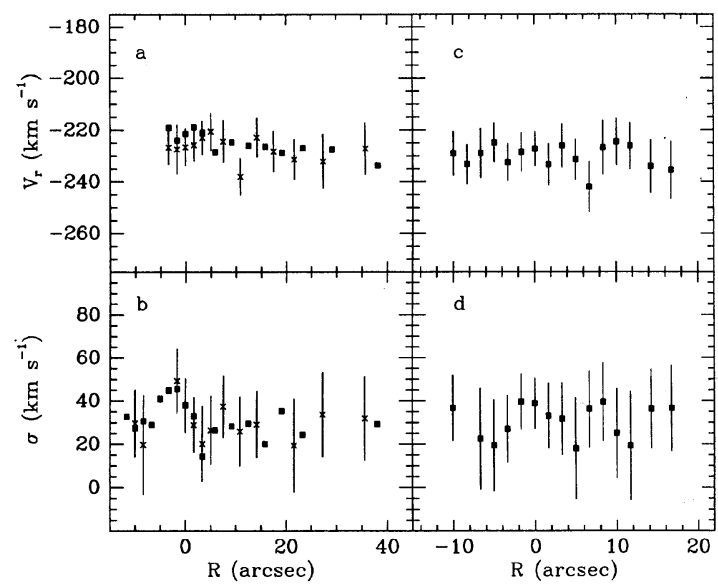

FIG. 1. The rotation curves and velocity dispersion profiles of NGC 185. (a) Heliocentric radial velocity profiles along the major axis. Filled squares refer to the sum of spectra taken at a position angle $230^{\circ}$; crosses refer to P.A. $=50^{\circ}$. (c) Velocity profile along the minor axis. (b),(d) Velocity dispersions measured along the major axis (b) and along the minor axis (d). Symbol coding as in (a). Error bars represent formal errors from the Fourier fitting technique. For clarity, they are drawn for one dataset only. The scale is $3.5 \mathrm{pc} \operatorname{arcsec}^{-1}$. 


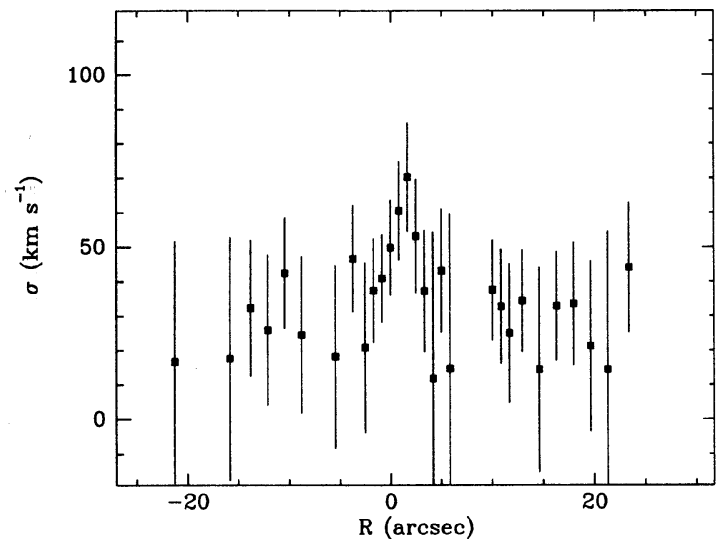

FIG. 2. The velocity dispersion profile in the central region of NGC 185 , as measured on the spectrum with spatial binning 0.836 arcsec pixel $^{-1}$.

by the Fourier program are plotted in Figs. 1 and 2 . The internal errors of the measurements can be judged also from the close agreement between the two datasets. Taking into account the results of the simulations, and the fact that the derived dispersion is (marginally) larger than $20 \mathrm{~km} \mathrm{~s}^{-1}$, we consider the kinematic profiles of NGC 185 to be reliable out to $40^{\prime \prime}$.

Bender et al. (1991) report major-axis observations of NGC 185 extending to $105^{\prime \prime}$. They find $\sigma=23 \pm 5 \mathrm{~km} \mathrm{~s}^{-1}$, and an upper limit to $v$ of only $3 \mathrm{~km} \mathrm{~s}^{-1}$. This is fully in accord with our observations. Their data do not show an increase of $\sigma$ in the inner few 2-3", but this may well be due to the fact that they used $5^{\prime \prime}$ bins.

The remarkably flat rotation curve in Fig. 1 suggests that NGC 185 is a further example of a nonrotating, pressuresupported dwarf elliptical galaxy. The parameter $v / \sigma$ (Binney 1978) provides a quantitative estimate of the relative dynamic importance of rotation and random motions. Its expected value $(v / \sigma)_{0}$ for rotation-supported oblate spheroids is approximately $\sqrt{\epsilon /(1-\epsilon)}$ (Kormendy 1982). With a mean ellipticity $\epsilon=0.23$ (computed within $30^{\prime \prime}$ from the center; Kent 1987), this gives $(v / \sigma)_{0}=0.55$ for NGC 185. Assuming a fairly conservative upper limit of $10 \mathrm{~km} \mathrm{~s}^{-1}$ on $v$, we find $(v / \sigma)=0.65 \times(v / \sigma)_{0}$. We take this result as evidence that rotation alone cannot account for the observed flattening of NGC 185. As was the case for NGC 205, this dwarf elliptical appears to be supported by an anisotropic velocity distribution, and may well be triaxial.

All of the dwarf ellipticals studied so far, and also the low-luminosity ellipticals close to the dwarf sequence, appear to be anisotropic pressure-supported ellipsoids (Bender \& Nieto 1990; Carter \& Sadler 1990; Paper I; Paltoglou \& Freeman 1987). This agrees with the suggestion by Wyse \& Jones (1984) that low-surface-brightness dwarf ellipticals would be slowly rotating objects. It may also be significant that the specific angular momentum of anisotropic low-luminosity ellipticals does not differ from that of rotation-supported objects of comparable luminosity (Bender \& Nieto 1990).

Observations of the $\mathrm{H}$ I component (Johnson \& Gottesman 1983) add some information on the kinematics of NGC 185. While a $5 \times 10^{5} \mathscr{M}_{\odot}$ cloud of $\mathrm{H}$ I was found at $30^{\prime \prime}$ from the nucleus, not far from the major axis, no $\mathrm{H} \mathrm{I}$ emission was detected from either a gaseous disk or the nucleus itself. The velocity distribution of this $\mathrm{H}$ I cloud reveals a clumpy structure. It is characterized by an asymmetric, double-peaked profile, with a principal peak corresponding to a radial velocity $-195 \mathrm{~km} \mathrm{~s}^{-1}$, and a much lower peak at -226 $\mathrm{km} \mathrm{s}^{-1}$. The former peak differs significantly from the optical redshift. The uncertainties in the optical central velocity, however, make it difficult to establish whether the principal $\mathrm{H}$ I cloud is in circular motion around the center of NGC 185. The emission profile of the molecular gas observed by Wiklind \& Rydbeck (1986) has two peaks, centered at the velocities -292 and $-203 \mathrm{~km} \mathrm{~s}^{-1}$. Only this latter peak does not differ too much from the systemic velocity, and might be associated with an ordered rotation.

Given the limited spatial extent of our kinematic profiles, we did not attempt any fitting of detailed dynamical models. Binney's (1982) formula for an isotropic spherical galaxy with a de Vaucouleurs' profile gives a mass-to-light ratio $\mathscr{M} / L_{B}$ for the central region of NGC 185 equal to 3.9 in solar units. Taking into account the effects of oblateness and anisotropy by means of the method of Bacon et al. (1985) lowers this value to 3.3. Since the luminosity profile of NGC 185 is nearly exponential, we computed the properties of an isotropic spherical model with an exponential projected surface brightness profile. Following Binney's (1982) method, this results in an expression for $\mathscr{M} / L_{B}$ which is identical to his Eq. (20), except for the numerical factor, which is now 0.172 rather than 0.201 . This implies a $15 \%$ decrease in the computed mass-to-light ratio with respect to $r^{1 / 4}$ spherical models. We conclude therefore that the central $\mathscr{M} / L_{B}$ in NGC 185 is about 3.

This rather low value of $\mathscr{M} / L_{B}$ is not unusual compared with the values found for the inner regions of giant elliptical galaxies (e.g., Kent 1990), but higher than those of Galactic globular clusters, which generally lie between 1 and 2 (Pryor et al. 1988, 1989). We note that our earlier value of $\mathscr{M} / L_{B}=7$ for NGC 205 (Paper I) was based on $\sigma \simeq 60$ $\mathrm{km} \mathrm{s}^{-1}$. If we take the value of $42 \mathrm{~km} \mathrm{~s}^{-1}$ reported by BPN, then $\mathscr{M} / L_{B}$ for NGC 205 comes down to 3.5 .

Our kinematic profiles for NGC 185 cover a region which is small compared to the galaxy size: they extend out to $\sim 1 / 5$ of the effective radius $r_{e}$. Our dynamical conclusions therefore strictly apply only to the central region. The data of BPN reduce our ignorance, but their kinematic curves still reach only $0.5 r_{e}$. As a result, we do not know the maximum rotation velocity, or the velocity dispersion, at radii comparable with those providing evidence for dark matter in dwarf irregulars (Carignan et al. 1990; Lake et al. 1990; and references therein).

\section{4. $L$ vs $\sigma$ : BRIDGING THE GAP}

The relations between kinematic and structural properties of normal elliptical galaxies have proven to be essential tools for understanding their origin and evolution. Although it has been demonstrated that elliptical galaxies are better described as a two-parameter family (see Kormendy \& Djorgovski 1989 for a comprehensive review), the relation between velocity dispersion and luminosity (Faber \& Jackson 1976) still represents a simple and well-defined constraint for models of galaxy formation. Analyzing the $L-\sigma$ relation for dwarfs is certainly only a first step toward a more complete (multiparametric) investigation. However, it is intended to provide some physical insight through a direct comparison with the predictions of published models. Until 
recently, no kinematic data were available for dwarf ellipticals in the intermediate luminosity range -18 $<M_{B}<-14$. The new data prompted us to attempt a reanalysis of the correlations between photometric and kinematic properties for spheroidal systems. Velocity dispersions have been collected for globular clusters and dwarf spheroidals in the Local Group, and for luminous ellipticals. They have been plotted against absolute magnitude $\mathscr{M}_{B}$ in Fig. 3.

Kinematic profiles derived from Fourier fitting of absorption features in integrated spectra are available for three of the dwarf companion galaxies to M31: NGC 205 (Carter \& Sadler 1990; Paper I), NGC 185 (this work; BPN), and NGC 147 (BPN). The recent paper of BPN presents new observations of NGC 147. Its brightness and velocity dispersion are nearly identical to those of NGC 185 . Velocity dispersions are available also for two dwarf ellipticals beyond the Local Group. In the sample of low-luminosity ellipticals studied by Bender \& Nieto (1990), one galaxy has sufficiently low surface brightness to be positively classified as a dwarf elliptical (IC 794; Binggeli et al. 1985). The classification of another object in the same sample (VCC 351) is controversial because of its relativèly high surface brightness, and we exclude it from our discussion. Lastly Bothun et al. (1985) measured the central velocity dispersion for the strong-lined nucleated dwarf elliptical VCC 1389 in the Virgo cluster. Absolute magnitudes for the companions to M31 were computed from total magnitudes in the RC2 (de Vaucouleurs $e t$ al. 1976) for a distance of $720 \mathrm{kpc}$. Reddening corrections are uncertain because of the low galactic latitude and patchy gas and dust distribution toward M31. The luminosity of IC 794 was adapted from Bender \& Nieto (1990), with $H_{0}=75$ $\mathrm{km} \mathrm{s}^{-1} \mathrm{Mpc}^{-1}$, while the VCC 1389 blue magnitude was taken from Ichikawa et al. (1986), assuming a Virgo distance modulus of 31 .

Internal velocity dispersions of dwarf spheroidals in the Local Group were taken from Aaronson's (1986) review (Dra, UMi, and Scl), Zaritsky et al. 1989 (Leo I and Leo II), and Godwin \& Lynden-Bell (1987) (Car). Leo I has no detected internal dispersion (after correction for measurements errors). A mean value of $6 \mathrm{~km} \mathrm{~s}^{-1}$ was adopted for Fornax based on the recent observations by Rodgers \& Harding (1989). ${ }^{1}$ Note that the velocity dispersions of dwarf spheroidals are subject to significant observational uncertainties. Difficulties related to radial velocity measurements of individual $\mathbf{C}$ and $\mathrm{K}$ giants, such as the effects of atmospheric motions, tidal disruption, binarity, and selfgravitation, are discussed by several authors (e.g., Aaronson \& Olszewski 1987). Moreover, velocity dispersions generally are computed with different procedures. Some of them are uncorrected measurements, while in other cases values corrected for the contribution of observational errors are given. In addition, even corrected dispersions may be overestimated. By comparing velocities obtained by different authors for the same stars in Carina, Godwin \& Lynden-Bell (1987) have demonstrated that they are not correlated. Thus for all spheroidals, the contribution of measurement errors to velocity dispersions might be substantial.

Absolute magnitudes of the dwarf spheroidals reported in the literature are also uncertain indirect estimates. We derived $M_{B}$ from $V$ magnitudes given by Aaronson (1986), assuming a constant $(B-V)=0.7$.

'The higher value of $9.9 \mathrm{~km} \mathrm{~s}^{-1}$ recently measured by Mateo et al. (1991b) does not affect the conclusions of this paper.

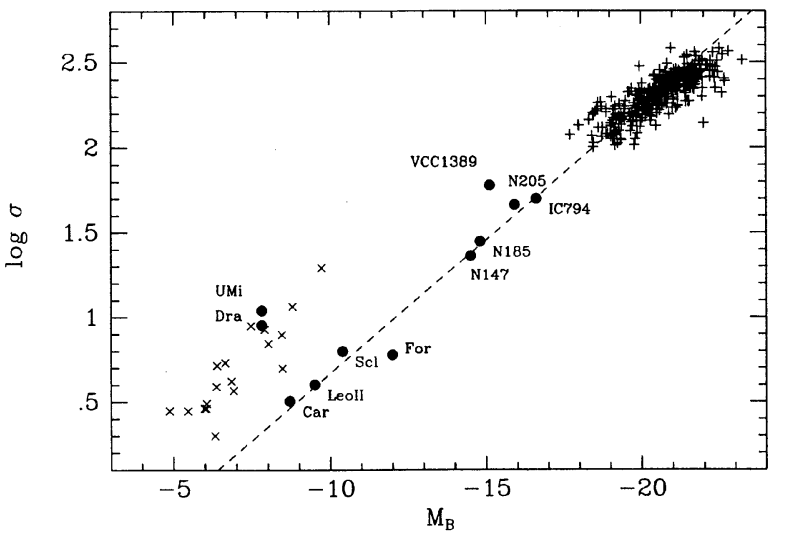

FIG. 3. Velocity dispersion $\sigma$ against absolute magnitude $M_{B}$, for dwarf elliptical and spheroidal galaxies (filled dots), normal ellipticals (plus signs), and globular clusters (crosses). The dashed line represents a fit to Car, Leo II, Scl, For, NGC 185, NGC 205, NGC 147, and IC 794, and corresponds to the approximate relation $L \propto \sigma^{2.5}$.

For comparison purposes, data for Galactic globular clusters are also shown in Fig. 3. Sources of velocity dispersions are the following: Da Costa et al. (1977); Gunn \& Griffin (1979); Lupton et al. (1987); Meylan \& Mayor (1986); Peterson \& Latham (1986); Pryor et al. (1986, 1988). In all of these studies, accurate central velocity dispersions were derived by fitting a dynamical model to velocities of individual giant stars. Illingworth (1976) measured internal velocity dispersions of globular clusters from their integrated spectra. Since his results tend to yield systematically higher dispersions than provided by measurement of individual giants, they have not been included in Fig. 3. Absolute magnitudes $M_{V}$ of Galactic globulars, adopted from Webbink's (1985) compilation, were converted into $B$ magnitudes using colors of Reed (1985) and Webbink's reddening values.

Finally, velocity dispersions and luminosities of normal elliptical galaxies have been taken from Faber et al. (1989), again assuming a Hubble constant $H_{0}=75$ $\mathrm{km} \mathrm{s}^{-1} \mathrm{Mpc}^{-1}$. As we are only interested in the average location of normal ellipticals in the luminosity-dispersion plane, the model dependence of the Faber et al. distances is of no concern here.

Figure 3 shows that most data points for dwarf elliptical galaxies define a linear sequence in the $\left(\log \sigma, M_{B}\right)$ diagram, extending continuously the $F-J$ relation for normal ellipticals. The two faintest dwarfs, Draco and Ursa Minor, represent an obvious exception to this trend. They are also the only dwarf spheroidals for which very high $\mathscr{M} / L$ ratios and central densities of dark matter have been inferred (Aaronson \& Olszewski 1987; Lake 1990; but see Kuhn \& Miller 1989 for a different view). In this paper, we restrict our discussion to the sequence of dE's brighter than $M_{B}=-8$. Dwarf ellipticals depart from a least-squares linear fit to $\mathrm{gE}$ galaxies, in the sense that dwarfs define a steeper slope. To quantify this trend we have fitted a straight line to the data for eight dwarfs in the luminosity range $-17<M_{B}<-8$. We have minimized the squared distances from the fitting line rather than the residuals in either $\log \sigma$ or $M_{B}$; all points are given equal weight. We have excluded VCC 1389 from 
the fit, because of the large uncertainty in its velocity dispersion.

Formally, the derived slope corresponds to a relation $L \propto \sigma^{2.5}$, which is indicated by the dashed line in Fig. 3. For comparison, a straight line fitted to dispersions and luminosities of globular clusters and ellipticals yields an exponent $k=3.3$.

The power-law index 2.5 is in close agreement with the slope predicted by models of formation of dwarf elliptical galaxies with early supernova-driven galactic winds and adiabatic expansion (Saito 1979; Dekel \& Silk 1986; Vader 1986, 1987; Yoshii \& Arimoto 1987, hereafter referred to as YA; Angeletti \& Giannone 1990). The YA population synthesis models include galactic mass loss and predict the structural and chemical properties of spheroidal systems based on simple assumptions concerning the star formation rate and the initial mass function. They contain no dark matter apart from stellar remnants. The fractional amount of gas lost by the system is highest for models in the range of initial masses appropriate for dwarfs $\left(10^{7-9} \mathscr{M}_{\odot}\right)$. Models with slow gas removal and adiabatic expansion are consistent with the observed distribution of dwarf ellipticals in the diameter versus surface-brightness diagram. For these models, the expected relation between velocity dispersion and luminosity (YA, Fig. 10) deviates slightly from the $L \propto \sigma^{4}$ line, in the sense of lower dispersions for fainter galaxies. With a smooth transition from giant to dwarf ellipticals, the dispersion versus luminosity relation steepens at the faint end $\left(M_{B} \sim-18\right)$ of the normal elliptical sequence, consistent with observations (Tonry 1981; Davies et al. 1983), and dwarf ellipticals in the range $-14<M_{B}<-18$ approximately follow the relation $L \propto \sigma^{2.5}$. This prediction is entirely consistent with the observational trend found in this paper.

A similar relation $L \propto \sigma^{2.7}$ for dwarf ellipticals was predicted also just on the basis of the observed correlation $L \propto r_{e}^{4}$ between effective radius and luminosity, under the simple assumptions that dE's have (as a class) a nearly constant $M / L$ and are in virial equilibrium (Binggeli et al. 1984; selfgravitating models in Dekel \& Silk 1986).

As pointed out by Vader (1986) and Dekel \& Silk (1986), simple self-gravitating gas-loss models seem to be unable to reproduce the observed correlation between mean metallicity $z$ and luminosity, $L \propto z^{2.5}$ (Vigroux et al. 1981; Aaronson \& Mould 1985; Buonanno et al. 1985; but see Mateo et al. 1991a for the case of Sextans). In particular, they apparently cannot account for the low metallicities of dwarf spheroidals (but see Vader 1986, 1987; YA). A dark matter scenario in which dwarf proto-galaxies are embedded in a dominant dark halo (Dekel \& Silk 1986) yields a metallicity-luminosity relation as required by the observations. In this case, however, a relation $L \propto \sigma^{5.3}$ is obtained which is inconsistent with the data for dwarf ellipticals brighter than $M_{B}=-8$. Thus, dark matter is probably not the key ingredient of the solution. That is not to say that the luminous matter we are able to study is not enclosed in such a halo, as is well demonstrated in the case of $\mathrm{gE}$ galaxies (Fabricant \& Gorenstein 1983; Mould et al. 1990).

Figure 3 does not show any evidence for a discontinuity in the global kinematic properties of dwarf and normal ellipticals. Whether the families of gE's and dE's form a continuous sequence of physical properties is a much debated issue (e.g., Kormendy \& Djorgovski 1989, and references therein). Kormendy (1985), using core radius and central surface brightness, showed that known correlations between structural parameters for dwarf and normal ellipticals define two distinct, apparently unrelated sequences. This discontinuity was taken to be evidence for very different formation processes. On the other hand, when similar correlations are investigated using global parameters (effective radius and surface brightness), giant and dwarf ellipticals fall on a continuous sequence, with the location along the sequence being determined by mass-dependent effects of gas loss (Binggeli et al. 1984, Figs. 7 and 8; Ferguson \& Sandage 1988, Figs. 5 and 6; YA; Nieto 1990).

It is of interest to compare the $\log r_{c}-M_{B}$ and $\log r_{e}$ $-M_{B}$ correlations (or their analogs for surface brightness) obtained by Kormendy (1985) and Binggeli et al. (1984), respectively. While the sequence of dwarf ellipticals is shifted to larger radii with respect to gE's in the (core radius, luminosity) diagram, the trend is the same whether core or global parameters are used. Cross examination of the surface brightness-luminosity relations leads to a similar remark. There is a simple explanation for the sharp discontinuity found when using core properties. Dwarf ellipticals generally have exponential luminosity profiles, in contrast to the $r^{1 / 4}$ de Vaucouleurs' profiles of gE's. If two model galaxies with an exponential and an $r^{1 / 4}$ profile are constrained to have the same luminosity $L$, then

$$
r_{e, d V}^{2} I_{0, d V} \simeq 210 \times r_{e, \exp }^{2} I_{0, \exp },
$$

with $r_{e, \exp }, r_{e, d V}$ the half-luminosity radii, and $I_{0, \exp }, I_{0, d V}$ the central surface brightnesses of the two profiles, respectively. Binggeli et al. (1984) show that dE's and gE's have comparable values of the model-independent effective radius, and therefore of the mean surface brightness inside $r_{e}$, in the overlap region $M_{B} \sim-20$. For the model central surface brightness, this implies $\mu_{0, \exp }-\mu_{0, d V} \simeq 5.8$. While the exact value of this difference depends on the real core profiles of $E$ galaxies (luminous ellipticals have larger core radii than would be predicted by a seeing-convolved $r^{1 / 4}$ profile; Kormendy 1985) and on model-dependent seeing corrections, its order of magnitude is entirely consistent with the shift of the dE sequence in the $\left(M_{B}, \mu_{0 V}\right)$ plane (Fig. 3 of Kormendy 1985). We thus conclude that the location of dwarf ellipticals in this plane only reflects their well-known prevailing exponential profiles. This can also explain the $\left(M_{B}, r_{c}\right)$ relation: it is easy to show that $r_{c, \exp } \gg r_{c, d V}$ for exponential and $r^{1 / 4}$ galaxies with the same luminosity and effective scale length.

As mentioned in Sec. 3, at least for isotropic spherical models the derived $\mathscr{M} / L$ value depends little on the details of the luminosity profile. To the extent that $\mathscr{M} / L$ is only moderately affected by anisotropy, the fact that dE's and gE's show similar values of $r_{e}$ and $\sigma$ around $M_{B} \sim-20$ may have an important consequence. At least in the luminosity range where the two families overlap, dE's and gE's may have comparable $\mathscr{M} / L$ values.

In summary, dwarf and normal ellipticals show no discontinuity in their global dynamical and photometric properties. The discontinuity in the relations for core photometric parameters merely exhibits quantitatively that dE's are much more diffuse than gE's. On the other hand, our results for NGC 185 seem to confirm that dwarf ellipticals are anisotropic objects. This fact, the different luminosity profiles, and the different trends of the photometric relations all indicate that dE's and gE's form two different families. Does this imply that they are also completely different in origin? If the 
galactic wind picture is correct, there may be a continuous transition, the key being the fractional amount of gas available (and lost) at the epoch of the onset of galactic winds (YA). Galactic wind models offer an attractive qualitative picture to account for the shallow luminosity distribution of dwarf ellipticals. Detailed quantitative models will be required, however, to explain how $\mathrm{dE}$ protogalaxies, following the gas loss and expansion process, may also become nonrotating anisotropic objects with nearly exponential luminosity profiles. Further kinematic data for dwarf ellipticals and spheroidals will provide more stringent constraints on models of galaxy formation and evolution. This is an appropriate task for the new generation of very large telescopes.

We thank M. Franx for allowing us to use his software, M. Franx and S. Djorgovski for useful comments, and L. Ciotti for helpful conversations. We also thank the referee for constructive comments. Partial support for this project was provided by NSF Grant No. AST-87-21705.

\section{REFERENCES}

Aaronson, M. 1986, in Stellar Populations, edited by C. Norman, A. Renzini, and M. Tosi (Cambridge University, Cambridge), p. 45.

Aaronson, M., \& Mould, J. R. 1985, ApJ, 290, 191

Aaronson, M., \& Olszewski, E. 1987, in Dark Matter in the Universe, IAU Symposium No. 117, edited by J. Kormendy and G. R. Knapp (Reidel, Dordrecht), p. 153

Angeletti, L., \& Giannone, P. 1990, A\&A, 234, 53

Bacon, R., Monnet, G., \& Simien, F. 1985, A\&A, 152, 315

Bender, R., \& Nieto, J.-L. 1990, A\&A, 239, 97

Bender, R., Paquet, A., \& Nieto, J.-L. 1991, A\&A, 246, 349

Binggeli, B., Sandage, A., \& Tarenghi, M. 1984, AJ, 89, 64

Binggeli, B., Sandage, A., \& Tammann, G. A. 1985, AJ, 90, 1681

Binney, J. J. 1978, MNRAS, 183, 501

Binney, J. J. 1982, ARA\&A, 20, 399

Bothun, G. D., Mould, J. R., Wirth, A., \& Caldwell, N. 1985, AJ, 90, 697

Buonanno, R., Corsi, C. E., Fusi Pecci, F., Hardy, E., \& Zinn, R. 1985,

A\&A, 152, 65

Carignan, C., Beaulieu, S., \& Freeman, K. C. 1990, AJ, 99, 178

Carter, D., \& Sadler, E. M. 1990, MNRAS, 245, 12P

Da Costa, G. S., Freeman, K. C., Kalnajs, A. J., Rodgers, A. W., \& Stapinski, T. E. $1977, \mathrm{AJ}, 82,810$

Davies, R. L., Efstathiou, G., Fall, S. M., Illingworth, G. D., \& Schechter, P. L. 1983, ApJ, 266, 41

Dekel, A., \& Silk, J. 1986, ApJ, 303, 39

de Vaucouleurs, G., de Vaucouleurs, A., \& Corwin, H. G. 1976, Second Reference Catalog of Bright Galaxies (University of Texas, Austin) (RC2)

Faber, S. M., \& Jackson, R. E. 1976, ApJ, 204, 668

Faber, S. M., Wegner, G., Burstein, D., Davies, R. L., Dressler, A., LyndenBell, D., \& Terlevich, R. J. 1989, ApJS, 69, 763

Fabricant, D., \& Gorenstein, P. 1983, ApJ, 267, 535

Ferguson, H. C., \& Sandage, A. 1988, AJ, 96, 1520

Franx, M., Illingworth, G. D., \& Heckman, T. M. 1989, ApJ, 344, 613

Godwin, P. J., \& Lynden-Bell, D. 1987, MNRAS, 229, 7P

Gunn, J. E., \& Griffin, R. F. 1979, AJ, 84, 752

Held, E. V., Mould, J. R., \& de Zeeuw, P. T. 1990, AJ, 100, 415 (Paper I)

Hodge, P. W. 1963, AJ, 68, 691

Ichikawa, S., Wakamatsu, K., \& Okamura, S. 1986, ApJS, 60, 475

Illingworth, G. 1976, ApJ, 204, 73

Johnson, D. W., \& Gottesmann, S. T. 1983, ApJ, 275, 549

Kent, S. M. 1987, AJ, 94, 306

Kent, S. M. 1990, in ASP Conference Series Vol. 10: Evolution of the Universe of Galaxies, edited by R. G. Kron (Astronomical Society of the Pacific, San Francisco), p. 109

Kormendy, J. 1982, in Morphology and Dynamics of Galaxies, edited by L.
Martinet and M. Mayor (Geneva Obs., Sauverny), p. 113

Kormendy, J. 1985, ApJ, 295, 73

Kormendy, J., \& Djorgovski, S. 1989, ARA\&A, 27, 235

Kuhn, J. R., \& Miller, R. H. 1989, ApJ, 341, L41

Lake, G. 1990, MNRAS, 244, 701

Lake, G., Schommer, R. A., \& van Gorkom, J. H. 1990, AJ, 99, 547

Lupton, R. H., Gunn, J. E., \& Griffin, R. F. 1987, AJ, 93, 1114

Mateo, M., Nemec, J., Irwin, M., \& McMahon, R. 1991a, AJ, 101, 892

Mateo, M., Olszewski, E., Welch, D. L., Fischer, P., \& Kunkel, W. 1991b, AJ, 102, 914

Meylan, G., \& Mayor, M. 1986, A\&A, 166, 122

Mould, J., Oke, J. B., de Zeeuw, P. T., \& Nemec, J. 1990, AJ, 99, 1823

Nieto, J.-L. 1990, in Dynamics and Interactions of Galaxies, edited by $R$ Wielen (Springer, Heidelberg), p. 258

Oke, J. B., \& Gunn, J. 1982, PASP, 94, 586

Paltoglou, G., \& Freeman, K. C. 1987, in Structure and Dynamics of Elliptical Galaxies, IAU Symposium No. 127, edited by P. T. de Zeeuw (Reidel, Dordrecht), p. 447

Peterson, R. C., \& Latham, D. W. 1986, ApJ, 305, 645

Price, J. S. 1985, ApJ, 297, 652

Pryor, C., McClure, R. D., Fletcher, J. M., Hartwick, F. D. A., \& Kormendy, J. 1986, AJ, 91, 546

Pryor, C., McClure, R. D., Fletcher, J. M., \& Hesser, J. E. 1988, in Globular Cluster Systems in Galaxies, IAU Symposium No. 126, edited by J. Grindlay and A. G. D. Philip (Kluwer, Dordrecht), p. 661

Pryor, C., McClure, R. D., Fletcher, J. M., \& Hesser, J. E. 1989, AJ, 98, 596

Reed, B. C. 1985, PASP, 97, 120

Rodgers, A. W., \& Harding, P. 1989, PASP, 101, 563

Saito, M. 1979, PASJ, 31, 193

Sandage, A. J., \& Tammann, G. A. 1981, A Revised Shapley-Ames Catalog of Bright Galaxies (Carnegie Institution of Washington, Washington, DC)

Tonry, J. L. 1981, ApJ, 251, L1

Vader, J. P. 1986, ApJ, 305, 669

Vader, J. P. 1987, ApJ, 317, 128

Vigroux, L., Chièze, J. P., \& Lazareff, B. 1981, A\&A, 98, 119

Webbink, R. F. 1985, in Dynamics of Star Clusters, IAU Symposium No.

113, edited by J. Goodman and P. Hut (Reidel, Dordrecht), p. 541

Wiklind, T., \& Rydbeck, G. 1986, A\&A, 164, L22

Wyse, R. F. G., \& Jones, B. J. T. 1984, ApJ, 286, 88

Yoshii, Y., \& Arimoto, N. 1987, A\&A, 188, 13

Zaritsky, D., Olszewski, E. W., Schommer, R. A., Peterson, R. C., \& Aaronson, M. 1989, ApJ, 345, 759 